\title{
Prospective Study
}

\section{Prevalence and Influence of Diagnostic Criteria in the Assessment of Hypogonadism in Intrathecal Opioid Therapy Patients}

Rui V. Duarte, PhD 1,2, Jon H. Raphael, MD 1,2, Mourad Labib, FRCPath ${ }^{3}$, Jane L. Southall, RGN² and Robert L. Ashford, $\mathrm{PhD}^{1}$

\begin{abstract}
From: ${ }^{1}$ Birmingham City University, Birmingham, UK; and ${ }^{2,3}$ Russells Hall Hospital, Dudley, UK

Dr. Duarte and Dr. Raphael are with the Faculty of Health, Birmingham City University, Birmingham, UK; and with the Department of Pain Management, Russells Hall Hospital, Dudley, UK Dr. Labib is with the Department of Clinical Biochemistry, Russells Hall Hospital, Dudley, UK Ms. Southall is with the Department of Pain Management, Russells Hall Hospital, Dudley, UK

Dr. Ashford is with the Faculty of Health, Birmingham City University, Birmingham, UK

Address Correspondence: Rui V. Duarte, PhD Birmingham City University Faculty of Health Westbourne Road Room 220, Ravensbury House Birmingham, B15 3TN UK E-mail: ruivduarte@gmail.com

Disclaimer: There was no external funding in the preparation of this manuscript.

Conflict of interest: None.

Manuscript received: 09-07-2012 Accepted for publication: 09-27-2012

Free full manuscript: www.painphysicianjournal.com
\end{abstract}

Background: Hypogonadism is frequently diagnosed based on total testosterone (TT) levels alone. However, $99 \%$ of testosterone is bound to the sex hormone-binding globulin (SHBG) with only $1 \%$ free testosterone. Alternative assessment methods consist of assay of free testosterone (FT) or bioavailable testosterone (BT) by equilibrium dialysis, calculation of FT and $\mathrm{BT}$ through the Vermeulen equations, and calculation of the free androgen index (FAI).

Objectives: The aim of this study was to investigate the prevalence of hypogonadism in male chronic non-cancer pain patients undertaking long-term intrathecal opioid therapy and the existence of diagnostic discrepancies according to the criteria used.

Study design: Prospective observational study.

Setting: Department of Pain Management, Russells Hall Hospital, Dudley, United Kingdom.

Methods: Twenty consecutive male patients undertaking long-term intrathecal opioid therapy had the gonadal axis evaluated by assays of luteinising hormone (LH), follicle stimulating hormone (FSH), TT, SHBG and by calculating the FT, BT and FAI.

Results: Hypogonadism was present in 17 (85\%) of the patients based on TT; 17 (85\%) according to FT and BT calculations; and 14 (70\%) when calculating FAI. Based on either TT or FT being low or borderline/low, 19 (95\%) of the investigated patients were biochemically hypogonadal. Significant differences were observed between diagnosis based on FT and FAI $(P<0.05)$. No significant differences were observed between diagnosis based on TT and FT $(P=0.40)$ or TT and FAI $(P=0.20)$.

Conclusion: Hypogonadism is common in patients undertaking intrathecal opioid therapy for the management of chronic non-malignant pain; however, diagnostic criteria can influence the diagnosis of this side effect. The assessment of the hypothalamic-pituitarygonadal axis should include evaluation of total serum testosterone, free testosterone, or bioavailable testosterone.

Key words: Diagnostic criteria, free testosterone, hypogonadism, implantable drug delivery systems, total serum testosterone

Pain Physician 2013; 16:9-14 $\square$ estosterone circulates in plasma non-specifically bound to albumin, specifically bound to sex hormone binding globulin (SHBG), and only a small percentage of testosterone is unbound (1).
SHBG also binds to estradiol which is the predominant female sex hormone. The free and the non-specifically bound testosterone represent a small percentage of the hormone available; however, there is good 
evidence that this fraction reflects more accurately the clinical situation than total testosterone in plasma (1). The unbound or free testosterone (FT) is able to link to and activate a receptor. SHBG bound to testosterone will inhibit this action.

Opioids are one of the factors that can modulate the hypothalamic-pituitary-gonadal axis functioning at the hypothalamic or pituitary level, with the potential to cause central hypogonadism, also known as secondary hypogonadism or hypogonadotropic hypogonadism. Treatment strategies for the management of chronic pain start with the lowest risk and least invasive intervention and progress if a treatment is not effective. The administration of opioids via intrathecal drug delivery systems (IDDS) is a last resort treatment for the management of severe chronic pain due their invasive nature, concerns about long-term opioid use, and the possible complications related to the procedure. Despite high initial costs, IDDS is a cost-effective treatment for the management of chronic non-malignant pain $(2,3)$. IDDS consists of an implanted pump which delivers medication directly into the cerebrospinal fluid through a catheter. The administration of opioids into the intrathecal space means effects are achieved at lower doses than using the epidural route (4). Analgesic efficacy is maximized at lower doses as the drug is highly localized (5). Therefore only a small amount of the drug is systemically absorbed (6). This leads to a decrease in the possible opioid side-effects.

The potential effect of IDD on the endocrine system is one of the least noted and investigated sideeffects (7). Currently, opioid induced hypogonadism is under-recognised and undertreated (8). Some patients may attribute the signs and symptoms of hypogonadism such as decreased libido, tiredness, loss of muscle mass and strength, among others, to the chronic pain and its related conditions rather than the intraspinal medication $(7,9)$. The current limited clinical awareness of the opioid effects on the endocrine system, together with the lack of information on their long-term consequences, is likely to result in a lack of information provision to the patient when long-term opioid therapy is being considered (10).

The gold standard test for verification of the androgen status is an assay of FT or bioavailable testosterone (BT) by equilibrium dialysis; however, this technique is not practical for routine evaluations since it is time consuming, requires sophisticated equipment, the use of freshly purified radioactive tracers, several hours of dialysis to achieve equilibrium and a long counting time for radioactivity $(11,12)$. This procedure examines the levels of total testosterone, SHBG, and albumin, therefore, allowing a precise analysis of FT and BT values. An alternative to this method is the calculation of FT and BT through the Vermeulen equations (1). The calculated FT has been considered as a reliable index of unbound testosterone $(1,13,14)$. The free androgen index (FAl) is also often calculated to examine the presence of hypogonadism. This index takes into consideration testosterone and SHBG levels but fails to take variations in albumin into account. The validity of FAl as a reliable equivalent of $\mathrm{FT}$ has been questioned with studies considering FAl a poor index of FT $(1,15)$.

The aim of this study was to investigate the prevalence of hypogonadism in male chronic non-cancer pain patients undertaking long-term intrathecal opioid therapy and diagnostic discrepancies according to the criteria employed.

\section{Methods}

\section{Participants}

Twenty consecutive male patients undertaking long-term IDD therapy at Russells Hall Hospital for the management of chronic non-cancer pain were included in the study. Patients who had received testosterone supplementation within the previous 3 months were not included (16). The pain syndrome the majority of the participants experienced was nociceptive (58.8\%), while neuropathic pain was present in $5.9 \%$ of the patients and $35.3 \%$ suffered from mixed nociceptiveneuropathic pain. All the patients were receiving intrathecal opioids for the management of their pain. Intrathecal morphine was the only medication administered to $50 \%$ of the sample. In individual cases, other substances were added to the intrathecal medication, with combinations of morphine with bupivacaine $(12.5 \%)$, morphine with bupivacaine and clonidine $(25 \%)$, and morphine with bupivacaine and baclofen (12.5\%).

\section{Assessment of Sex Hormones}

Blood samples were collected between 8am and 11 am during a 7-month period (April to October 2010) as part of routine clinical care, for the measurement of serum luteinizing hormone (LH), follicle-stimulating hormone (FSH), total testosterone (TT) and SHBG. All blood assays were carried out by the Department of Clinical Biochemistry at Russells Hall Hospital, Dudley. $\mathrm{LH}, \mathrm{FSH}$ and TT were measured according to the manufacturer's instructions by immuno-enzymometric fluori- 
metric assay on the Tosoh AIA 2000 LA analyser (Tosoh Bioscience N.V., Tessenderlo, Belgium). The inter-assay imprecision (\%CV) quoted by the manufacturer was $2.6 \%$ for $\mathrm{LH}, 2.3 \%$ for $\mathrm{FSH}$, and $5.3 \%$ for testosterone. SHBG was measured according to the manufacturer's instructions by chemiluminescent immunometric assay on the Immulite $2000 \mathrm{XPi}$ analyser (Siemens Healthcare Diagnostics Ltd, Camberley, Surrey, UK). The inter-assay variability $(\% \mathrm{CV})$ for SHBG was $5 \%$. Calculations of FT and BT were also carried out (1). The quoted reference ranges were $\mathrm{LH}(2.2-13.3 \mathrm{IU} / \mathrm{L}), \mathrm{FSH}(1-7 \mathrm{IU} / \mathrm{L})$, and SHBG $(13-71 \mathrm{nmol} / \mathrm{L})$. Serum testosterone $(\mathrm{TT})<8 \mathrm{nmol} / \mathrm{L}$, FT $<180$ picomoles per liter ( $\mathrm{pmol} / \mathrm{L}), \mathrm{BT}<3.8 \mathrm{nmol} / \mathrm{L}$, and/ or FAI $<15.3$ were considered diagnostic of biochemical hypogonadism $(17,18)$. Serum TT $8-12 \mathrm{nmol} / \mathrm{L}$, FT 180 - $250 \mathrm{pmol} / \mathrm{L}$, or $\mathrm{BT} 3.8-6.1 \mathrm{nmol} / \mathrm{L}$ was considered as borderline/low (17).

\section{Data Analysis}

Kolmogorov-Smirnov test was performed to test distribution of numerical data, followed by the appropriate statistical tests. Associations between variables were investigated by Spearman's correlation coefficients. Analyses of categorical data were carried out through Fisher's exact test. Data is reported as median (minimum-maximum). Statistical significance was judged at $5 \%$ level. Statistical tests were performed using Predictive Analytics SoftWare (PASW) (version 18.0, SPSS Inc., Chicago, IL, USA).

\section{Results}

The sample consisted of 20 male IDDS patients with a median age at the time of blood collection of 58 years (47-69). The time from implantation of the IDD system to hormone assay was 100 months (15- 203) with an intrathecal opioid dose of $2.68 \mathrm{mg} /$ day (range 1- 9.7) (Table 1). The duration of pain prior to commencement of IDD was 9 years (range 3-35).

Based on TT, 17 ( $85 \%$ ) of the patients were hypogonadal with $12(60 \%)$ at less than $8 \mathrm{nmol} / \mathrm{L}$ and $5(25 \%)$ between 8 and $12 \mathrm{nmol} / \mathrm{L}$ (Fig. 1). Based on FT calculations, $17(85 \%)$ were hypogonadal with $15(75 \%)$ at less than $180 \mathrm{pmol} / \mathrm{L}$ and $2(10 \%)$ patients between 180 and $250 \mathrm{pmol} / \mathrm{L}$. The same outcome was observed for the analysis of BT calculations, therefore FT was considered for further statistical comparisons as the result would be the same if carried out with either FT or BT. Based on hypogonadism defined as $\mathrm{FAl}<15.3$ in men, $14(70 \%)$ patients were identified. Based on either TT or FT being low or borderline/low, 19 (95\%) of the inves-
Table 1. Sample characteristics

\begin{tabular}{|l|c|}
\hline Nr patients & 20 \\
\hline Age (years) & $58(47-69)$ \\
\hline IDDS duration (months) & $100(15-203)$ \\
\hline Opioid dose (mg/day) & $2.68(1-9.7)$ \\
\hline LH (IU/L) & $1.9(0.2-19.9)$ \\
\hline FSH (IU/L) & $5.3(0.3-23.9)$ \\
\hline SHBG (nmol/L) & $51(17-123)$ \\
\hline TT (nmol/L) & $4.95(1.2-18.8)$ \\
\hline FAI & $10.4(1.9-57.06)$ \\
\hline BT (nmol/L) & $1.62(0.33-7.7)$ \\
\hline FT (pmol/L) & $69.45(14-328)$ \\
\hline
\end{tabular}

Key: LH - luteinizing hormone; FSH - follicle stimulating hormone; SHBG - sex hormone binding globulin; TT - total testosterone; FAI - free androgen index; BT - bioavailable testosterone; FT - free testosterone

Statistics are presented as median (minimum-maximum)

tigated patients, were biochemically hypogonadal. In this sample FAI was highly correlated with FT $(r=0.929$, $P<0.01)$ and BT $(r=0.929, P<0.01)$ and less associated with testosterone $(r=0.701, P<0.01)$. FT $(r=0.866, P$ $<0.01)$ and $B T(r=0.866, P<0.01)$ were more strongly correlated with testosterone. To investigate potential age-related differences in the diagnosis of hypogonadism, 2 groups were created based on the median age ( $<58$ and $\geq 58$ years). No significant differences were observed between the age groups and diagnosis based on TT $(P=0.566)$, FT $(P=0.566)$ or FAI $(P=0.336)$.

Significant differences were observed between diagnosis based on FT and FAI $(P<0.05)$. No significant differences were observed between diagnosis based on TT and FT $(P=0.40)$ or TT and FAl $(P=0.20)$.

\section{Discussion}

Significant differences were observed in this study when the diagnoses of hypogonadism were compared based on calculated FT or BT and FAl. The prevalence of this complication based on diagnosis criteria ranged from $70 \%$ (14 patients) for FAI to $85 \%$ (17 patients) based on TT or FT. When both TT and FT were considered $95 \%$ (19 patients) were diagnosed as biochemically hypogonadal. The incidence of this side effect, although variable, is approximate across prior studies. The diagnostic criteria may account for much of this variation. The majority of studies investigating the 


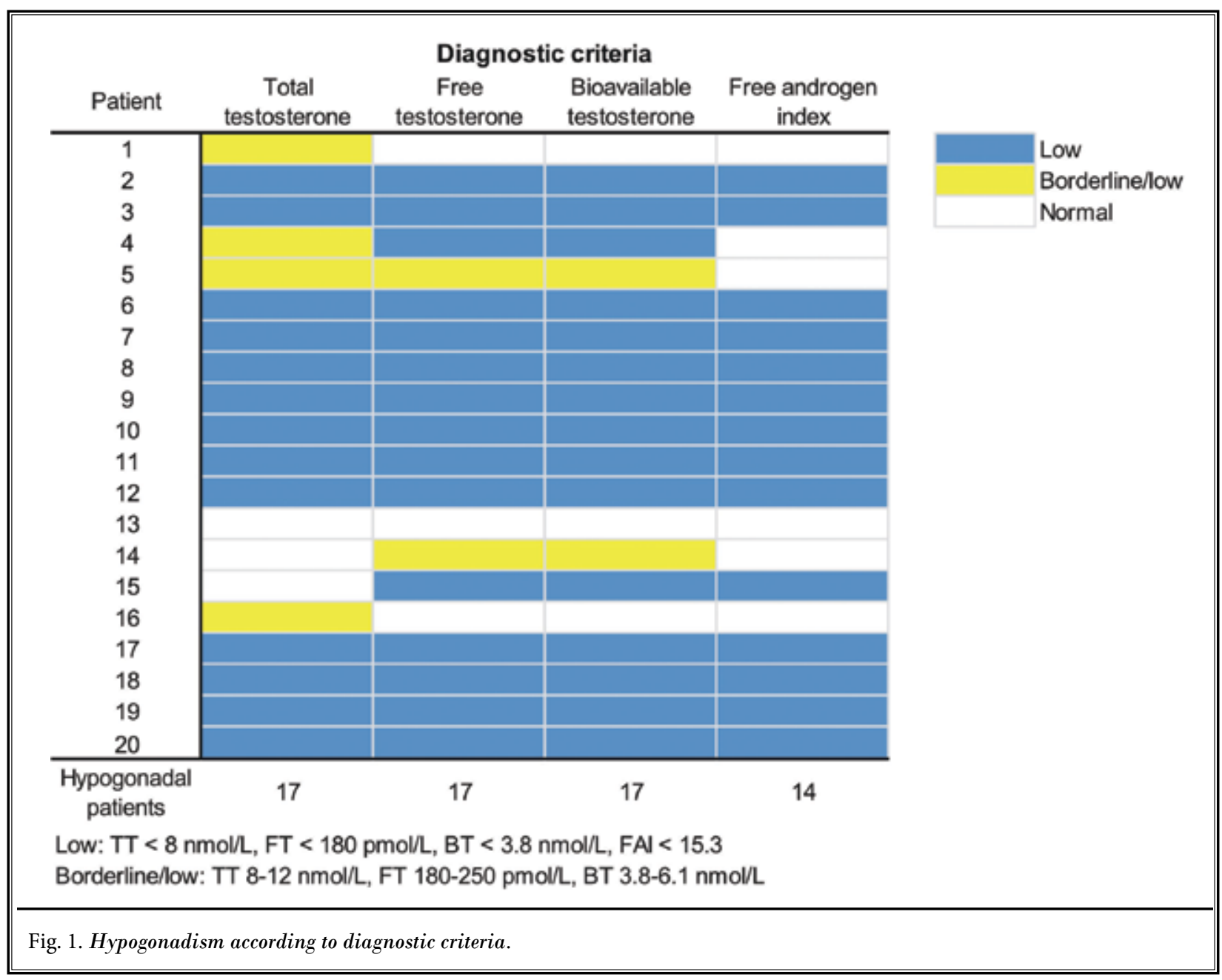

effects of intrathecal opioids in the endocrine system have considered mainly TT, LH, and FSH levels. To our knowledge, this is the first study with IDD patients that also used Vermeulens' calculated FT as a diagnostic tool. In studies where FAl was calculated, 25 of 29 intrathecal opioid patients $(86.2 \%)$ had a testosterone level lower than $9.0 \mathrm{nmol} / \mathrm{L}$ but only 18 of the patients $(62.1 \%)$ had a FAl lower than 20 (19). Another study reported that all the patients $(n=10)$ had testosterone below the normal range of 10 to $35 \mathrm{nmol} / \mathrm{L}$; however, the average $\mathrm{FAl}$ was $29.1 \pm 11.0$ (20). The prevalence of hypogonadism in male patients undertaking oral opioids has been reported to range from $83 \%$ to $90 \%(21-23)$. These results indicate a high prevalence of opioid induced hypogonadism, regardless of the administration route. Even at clinically very low intrathecal opioid doses $(1.5 \pm 0.7$ $\mathrm{mg} /$ day [range $0.35-2.3$ ]), hypogonadism was present in 5 out of 7 patients (24).

The development of hypogonadism is multifactorial and in this specific population there are many other possible confounding factors. Psychological aspects such as depression, passive coping strategies, and catastrophising are some of the factors hypothesized to influence the sexual function in chronic pain patients (25). Nevertheless, Monga et al (25) did not evaluate hormone levels in the studied sample. Chronic pain did not seem to be the cause of gonadal function reduction which was observed in patients undertaking intrathecal morphine therapy when comparing with a control group of chronic pain patients who were not taking any form of opioid drugs (20). Of the possible chronic illnesses identified in a longitudinal study with 890 male participants, only cancer (9\%) was associated with a greater decrease in testosterone levels than the 
decrease that occurred with aging alone (18).

The hypothalamic-pituitary-gonadal axis should be routinely monitored in IDD patients and adequate treatment provided. The patient may attribute signs and symptoms of hypogonadism to the chronic pain or feel embarrassed discussing this subject with the nurse or physician during the review clinics and although the practitioner should not rely on a number to diagnose this syndrome, biochemical assessment would be required prior to initiation of hormonal replacement therapy. The reliance on the patient to discuss hypogonadal symptoms can lead to an unawareness and underdiagnosis of this side effect in centers not monitoring the hypothalamic-pituitary-gonadal axis. Hypogonadism is a risk factor associated with several conditions. Low TT, FT, and SHBG are independent risk factors for later development of type 2 diabetes (26-27). Low testosterone levels may be associated with increased cardiovascular risk (13).

It is important to note the limitations of this study. A small number of patients were included without a control group and the gonadal status was not evaluated prior to commencement of IDD therapy. Women were not included in this study. Low libido and amenorrhea have been reported in female IDDS patients $(19,20,28)$, although the prevalence has been reported to be lower in women (22).

Opioid induced hypogonadism may be reversible. Clinically significant improvements in hypogonadal symptoms, sexual function, and mood were observed in men with opioid induced androgen deficiency being treated with transdermal testosterone patches (29). Improvements in aspects of sexual activity and decrease in ratings of mood aspects such as depression, anger, and fatigue were detected in previously untreated hypogonadal men following testosterone injections (30). Recovery of serum testosterone levels following cessation of IDD therapy or significant improvements in libido following hormonal replacement therapy have been reported $(19,20)$. After the opioid is stopped, testosterone levels generally recover within one month (31-33).

The increase of testosterone levels and consequently libido may have added importance to chronic pain patients. The results of animal studies have suggested that reduced libido may be linked with a lower pain threshold $(34,35)$. In humans, it has been observed that men with opioid-induced androgen deficiency required lower oral opioid doses during hormonal replacement therapy, suggesting that hypogonadism patients may have a lower pain threshold (21).

\section{Conclusion}

Hypogonadism is common in patients undertaking IDD therapy for the management of chronic non-malignant pain. Diagnostic criteria can influence the diagnosis of this side effect. The hypothalamic-pituitarygonadal axis should be routinely monitored in patients undertaking intrathecal opioid administration from the start of therapy. This assessment should include evaluation of total serum testosterone, FT, or BT. LH and FSH should also be measured to differentiate between primary or secondary hypogonadism.

\section{References}

1. Vermeulen A, Verdonck L, Kaufman JM. A critical evaluation of simple methods for the estimation of free testosterone in serum. J Clin Endocrinol Metab 1999; 84:3666-3672.

2. de Lissovoy G, Brown RE, Halpern M, Hassenbusch SJ, Ross E. Cost-effectiveness of long-term intrathecal morphine therapy for pain associated with failed back surgery syndrome. Clin Ther 1997; 19:96-112.

3. Biggs SA, Duarte RV, Raphael JH, Ashford RL. Influence of a latent period in QALY analysis: Pilot study of intrathecal drug delivery systems for chronic nonmalignant pain. $\mathrm{Br} J$ Neurosurg 2011; 25:401-406.
4. Nordberg G, Hedner T, Mellstrand T, Dahlström B. Pharmacokinetic aspects of intrathecal morphine analgesia. Anesthesiology 1984; 60:448-454.

5. Bernards C. Cerebrospinal fluid and spinal cord distribution of baclofen and bupivacaine during slow intrathecal infusion in pigs. Anesthesiology 2006; 105:169-178.

6. Winkelmüller $\mathrm{M}$, Winkelmüller W. Longterm effects of continuous intrathecal opioid treatment in chronic pain of nonmalignant etiology.] Neurosurg 1996; 85:458-467.

7. Doleys D, Dinoff B, Page L, Tutak U, Willis K, Coleton M. Sexual dysfunction and other side effects of intraspinal opiate use in the management of chronic noncancer pain. American Journal of Pain Management 1998; 8:5-11.

8. Reddy RG, Aung T, Karavitaki N, Wass $\mathrm{JAH}$. Opioid induced hypogonadism. BM] 2010; 341:C4462.

9. Katz N, Mazer NA. The impact of opioids on the endocrine system. Clin J Pain 2009; 25:170-175.

10. Vuong C, Van Uum SHM, O'Dell LE, Lutfy K, Friedman TC. The effects of opioids and opioid analogs on animal and human endocrine systems. Endocr Rev 2010; 31:98-132.

11. Raverot V, Lopez J, Grenot C, Pugeat M, Déchaud $H$. New approach for measurement of non-SHBG-bound tes- 
tosterone in human plasma. Anal Chim Acta 2010; 658:87-90.

12. Vermeulen A. Hormonal cut-offs of partial androgen deficiency: A survey of androgen assays. J Endocrinol Invest 2005; 28:28-31.

13. Hackett GI. Review: Androgens, erectile dysfunction and cardiovascular risk in type 2 diabetes. $\mathrm{Br}$ J Diabetes Vasc Dis 2009; 9:214-217.

14. Rosner W, Auchus RJ, Azziz R, Sluss PM, Raff $H$. Position statement: Utility, limitations, and pitfalls in measuring testosterone: An Endocrine Society position statement. J Clin Endocrinol Metab 2007; 92:405-413.

15. Kapoor P, Luttrell BM, Williams D. The free androgen index is not valid for adult males. J Steroid Biochem Mol Biol 1993; 45:325-326.

16. Roberts L, Finch P, Pullan P, Bhagat $C$, Price $L$. Sex hormone suppression by intrathecal opioids: A prospective study. Clin J Pain 2002; 18:144-148.

17. Hackett G, Cole NS, Deshpande AA, Popple MD, Kennedy D, Wilkinson P. Biochemical hypogonadism in men with type 2 diabetes in primary care practice. $\mathrm{Br} J$ Diabetes Vasc Dis 2009; 9:226-231.

18. Harman S, Metter E, Tobin J, Pearson J, Blackman M. Longitudinal effects of aging on serum total and free testosterone levels in healthy men. J Clin Endocrinol Metab 2001; 86:724-731.

19. Abs R, Verhelst J, Maeyaert J, Van Buyten $J P$, Opsomer F, Adriaensen $H$, Verlooy J, Van Havenbergh T, Smet V, Van Acker K. Endocrine consequences of long-term intrathecal administration of opioids.
J Clin Endocrinol Metab 2000; 85:22152222.

20. Finch P, Roberts L, Price L, Hadlow N, Pullan P. Hypogonadism in patients treated with intrathecal morphine. Clin J Pain 2000; 16:251-254.

21. Daniell HW. Hypogonadism in men consuming sustained-action oral opioids. J Pain 2002; 3:377-384.

22. Fraser LA, Morrison D, Morley-Forster P, Paul TL, Tokmakejian S, Nicholson LR, Bureau Y, Friedman TC, Van Uum $\mathrm{SH}$. Oral opioids for chronic non-cancer pain: Higher prevalence of hypogonadism in men than in women. Exp Clin Endocrinol Diabetes 2009; 117:38-43.

23. Rajagopal A, Vassilopoulou-Sellin R, Palmer J, Kaur G, Bruera E. Hypogonadism and sexual dysfunction in male cancer survivors receiving chronic opioid therapy. J Pain Symptom Manage 2003; 26:1055-1061.

24. Alam F, Duarte R, Raphael J, Labib M, Mutagi H, Kapur S. Low dose intrathecal morphine therapy and hypogonadotrophic hypogonadism. Reg Anesth Pain Med 2008; 33:e2O2.

25. Monga TN, Tan G, Ostermann HJ, Monga U, Grabois M. Sexuality and sexual adjustment of patients with chronic pain. Disabil Rehabil 1998; 20:317-329.

26. Feldman HA, Goldstein I, Hatzichristou DG, Krane RJ, McKinlay JB. Impotence and its medical and psychosocial correlates: Results of the Massachusetts Male Aging Study. J Urol 1994; 151:54-61.

27. Haffner SM, Shaten J, Stem MP, Smith GD, Kuller L; MRFIT Research Group. Low levels of sex hormone-binding globulin and testosterone predict the development of non-insulin-dependent diabetes mellitus in men. Am J Epidemiol 1996; 143:889-897.

28. Paice JA, Penn RD. Amenorrhea associated with intraspinal morphine. J Pain Symptom Manage 1995; 10:582-583.

29. Daniell HW, Lentz R, Mazer NA. Openlabel pilot study of testosterone patch therapy in men with opioid-induced androgen deficiency. J Pain 2006; 7:200210.

30. Burris AS, Banks SM, Carter CS, Davidson JM, Sherins RJ. A long-term, prospective study of the physiologic and behavioral effects of hormone replacement in untreated hypogonadal men. J Androl 1992; 13:297-304.

31. Cicero TJ, Bell RD, Wiest WG, Allison JH, Polakoski K, Robins E. Function of the male sex organs in heroin and methadone users. N Engl J Med 1975; 292:882887.

32. Mendelson JH, Mello NK. Plasma testosterone levels during chronic heroin use and protracted abstinence. A study of Hong Kong addicts. Clin Pharmacol Ther 1975; 17:529-533.

33. Wang C, Chan V, Yeung RT. The effect of heroin addiction on pituitary-testicular function. Clin Endocrinol 1978; 9:455-461.

34. Forman LJ, Tingle V, Estilow S, Cater J. The response to analgesia testing is affected by gonadal steroids in the rat. Life Sci 1989; 45:447-454.

35. Pednekar JR, Mulgaonker VK. Role of testosterone on pain threshold in rats. Indian J Physiol Pharmacol 1995; 39:423424. 